\title{
Characteristic of Hybrid Cellulose-Amino Functionalized POSS-Silica Nanocomposite and Antimicrobial Activity
}

\author{
Sivalingam Ramesh, Jaehwan Kim, and Joo-Hyung Kim \\ Laboratory of Intelligent of Devices and Thermal Control, Department of Mechanical Engineering, Inha University, \\ Inha-ro 100, Nam-gu, Incheon 402-751, Republic of Korea
}

Correspondence should be addressed to Joo-Hyung Kim; joohyung.kim@inha.ac.kr

Received 25 August 2014; Accepted 2 October 2014

Academic Editor: Cheol-Min Park

Copyright (C) 2015 Sivalingam Ramesh et al. This is an open access article distributed under the Creative Commons Attribution License, which permits unrestricted use, distribution, and reproduction in any medium, provided the original work is properly cited.

\begin{abstract}
Recently, cellulose has much attention as an emerging renewable nanomaterial which holds promising properties having unique piezoelectricity, insulating, and biodegradable nature for various applications. Also, the modified properties of cellulose by appropriate chemical modifications in various functional groups with outstanding properties or significantly improved physical, chemical, biological, and electronic properties will widen the way for it to be utilized in different usages. Therefore, in this paper, cellulose-functionalized polyhedral oligomeric silsesquioxanes (POSS) based materials were considered an important class of highperformance hybrid nanocomposite materials. To functionalize the regenerated cellulose, amino functionalized POSS material was synthesized via sol-gel covalent crosslinking process in presence of amino coupling agent. In this reaction, tetraethoxsilane (TEOS) and $\gamma$-aminopropyltriethoxy silane ( $\gamma$-APTES) as coupling agent for metal precursors were selected. The chemical structure of cellulose-amine functionalized bonding and covalent crosslinking hybrids was confirmed by FTIR and ${ }^{1} \mathrm{H}$ NMR spectral analysis. From the TEM results, well-dispersed hybrid cellulose-functionalized POSS-silica composites are observed. The resulting cellulose-POSS-silica hybrid nanocomposites materials provided significantly improved the optical transparency, and thermal and morphological properties to compare the cellulose-silica hybrid materials. Further, antimicrobial test against pathogenic bacteria was carried out.
\end{abstract}

\section{Introduction}

Cellulose is the primary component providing strength to wood and other plant structures. Recently, cellulose based smart material has received much attention due to its unique material nature for possible sensors, actuators, and electronic applications [1-3]. Similarly, silicon has been paid much attention of potential flexible electronics as an abundant semiconductor material for polymer functionality based reaction process $[4,5]$. For this reason, there are number of studies on synthesis of the cellulose-polymer composites to enhance the material performances such as thermal, mechanical, electrical, and morphological properties of the polymers [6-8]. Generally, the composite processes with metal oxide based materials are widely used in material design, fabrication, and applications in recent nanotechnologies [6-9]. It is reported that nanofibers and nanoporous materials have been used to obtain relatively large surface, unusual strength, high surface energy, and high surface activity compared to its volume ratio of existing material properties $[8,9]$.

In order to synthesize functional hybrid nanocomposites based on cellulose, gel process has a great potential for synthesis of polymer-metal oxide hybrid nanocomposites with high degree of homogeneity and purity of molecular level [10]. The sol-gel process has been already widely used in the area of mesoporous, membrane, and intelligent hybrid hydrogel materials $[9,10]$. The sol-gel reaction involves the hydrolysis of silica precursors and the condensation reaction of hydroxyl groups to form a hybrid nanostructure. One simple method is the mixing of organic compounds with a metal alkoxide, such as tetraethoxysilane (TEOS). During the sol-gel process the inorganic mineral is attached with in the organic matrix forming hydrogen bonding or covalent bonds between the organic phase and inorganic phase. Another one is the introduction of triethoxysilyl prophy amine group into the organic compounds prior to the sol-gel reactions with 
tetraethoxysilane (TEOS). Many applications, particularly in the biomedical applications, have been already studied for these kinds of hybrid nanomaterials. Mainly, the dispersion of inorganic metal oxides nanoparticles dispersed inorganic particles imparts or enhanced the material properties such as the hardness, brittleness, transparency, density, free volume, and thermal stability of polymer hybrids [11-13]. In addition, the polyhedral oligomeric silsesquioxanes (POSS), the smallest silica nanoparticles, have great attentions for smart materials properties. The POSS molecule, a class of compounds with inorganic silicon-oxygen core and bulky organic substituents on the outer surface, is a monodisperse, and well-defined cubic nanoparticle consisting of rigid structure and single crystal silica cores with eight corners [13-15]. Due to the availability of POSS molecules with a variety of both reactive and nonreactive substituent functionalities, the reactive functional groups of the polymer composites were synthesized using copolymerization, grafting, or blending methods $[14,15]$. The POSS reactive groups can be easily functionalized with a wide variety of functional organic groups such as amino, thiol, hydroxyl, and carboxylic acid groups functionalized into different polymerization process. Therefore, POSS and functionalized POSS nanocage molecules can play a significant role for the synthesis of novel hybrid materials for various chemical processes. The crystalline hybrid nanocomposites for highperformance electronic applications in this connection with synthesized POSS [16-18] are reported. In order to increase the possibility to use this metal oxides materials, the synthetic silicates are frequently subjected to surface modification process with different coupling agents. The surface properties are a crucial factor which characterizes the nanoparticles and some important parameters such as surface charge density, spherical interactions, and dispersive properties of colloid nanoparticles $[17,18]$. Based on these properties, organic-inorganic hybrid materials have been studied in past decades due to good, thermal, magnetic, optical, electronic, optoelectronic, mechanical, and biological properties [19, 20]. Therefore, the preparation of inorganic nanomaterials that possess desired properties is a great challenging task for hybrid nanocomposites. The cellulose molecular and nanosilicon dioxide particles with organic groups need to be further modified by sol-gel crosslinking process. Moreover, the new hybrid material of silica-cellulose with uniform sized, shape and composition has a feasibility to be used in wide applications such as catalysis and photonics [2123]. Also, the immobilization of certain strains of bacteria to withstand the effects of number of common antibiotics has led us to find novel strategies for the treatment of infections associated to antimicrobial properties and biomaterials [2024]. Therefore, the present study is focused on synthesis of cellulose-POSS-amine/silica hybrid nanomaterials by solgel method in presence of metal precursors with coupling agents. The chemical/physical structure and thermal, optical, and biological properties of cellulose-POSS-silica hybrid materials were characterized and explained in detail.

\section{Experimental}

2.1. Materials. The synthesis of POSS-amine is from starting precursor's materials: octaphenyl silsesquioxane (OPS), phenyl trichlorosilane, and benzyl trimethyl ammonium hydroxide ( $40 \%$ in methanol solution). The second step is for nitration reaction, stepwise amine formation of POSS compounds. N,N-dimethylacetamide (DMAc), N,Ndimethylsulfoxide (DMSO) triethylamine and some of coupling chemicals for sodium acetate were used for the synthesis of POSS materials. Synthesis of cellulose-POSS-silica hybrid materials requires cotton pulp (Buckeye), lithium chloride in presence of sulfuric acid process in solution, tetraethoxysilane (TEOS), $\gamma$-aminopropyl triethoxy silane ( $\gamma$-APTES), and hydrochloric acid $(\mathrm{HCl})$. The antimicrobial test with agar sol and pathogenic bacteria such as Bacillus cereus (F4810/72) and Escherichia coli (ATCC 35150) was carried out for antimicrobial test.

\subsection{Synthesis of Cellulose-Amino Functionalized POSS-} Silica. Figure 1 shows the detailed synthesis process of cellulose-POSS-silica nanocomposite materials. The amino-functionalized POSS materials were synthesized by three steps. In first step, the synthesis of octaphenyl silsesquioxane (OPS) from phenyl trichlorosilane $(66.13 \mathrm{~g}$, $100 \mathrm{~mL}, 0.313 \mathrm{~mol}$ ) was placed, along with $160 \mathrm{~mL}$ of benzene, in a $2.5 \mathrm{~L}$ three-neck, round-bottom flask fitted with a magnetic stirrer and a dropping funnel. The required amount of water $(330 \mathrm{~g})$ was added dropwise, and the reaction was carried out at room temperature during the overnight. Then, the reaction mixture was washed with water until it became neutral, and the aqueous layer was removed. After that, $15 \mathrm{~mL}$ of $40 \%$ methanolic benzyl trimethyl ammonium hydroxide was added to the organic layer, and the reaction mixture was refluxed during $5 \mathrm{hrs}$. Then, the reaction mixture was allowed to stand at room temperature for 4 days and was again refluxed for $24 \mathrm{hrs}$. A white, solid powder weight with a yield of $97.8 \%$ was obtained by filtration. The first product was dried in a vacuum oven at room temperature and then purified with ethanol solution. The hybrid formation is shown in Figure 1(a). In second step, the synthesis of octanitrophenyl silsesquioxane (ONPS) was synthesized by following the reported method [25]. The same three-neck round-bottom flask equipped with a mechanical stirrer, $180 \mathrm{~mL}$ of fuming nitric acid, was placed to the flask. The flask was cooled with ice bath conditions and then OPS ( $30 \mathrm{~g})$ were added in portions, and the flask was kept in an ice bath for about a $1 \mathrm{hr}$. Then, the reaction mixture was stirred during overnight at room temperature and the reaction mixture was poured on to ice cold conditions and kept for $4 \mathrm{hrs}$. The light yellow colored resulting product was filtered, washed with deionized water until the condition of $\mathrm{pH}=6$, and then washed with $120 \mathrm{~mL}$ of ethanol. Finally, resulting product was obtained for solid powder and airdried at room temperature during overnight. In third step, octa(aminophenyl) silsesquioxanes (OAPS) - the synthesis from ONPS $(5 \mathrm{~g}, 3.60 \mathrm{mmol})$ with $0.30 \mathrm{~g}(0.283 \mathrm{mmol})$ of 5 wt.\% palladium/carbon $(\mathrm{Pd} / \mathrm{C})$ - were placed in a threeneck, round-bottom flask with a magnetic stirrer. A steady 


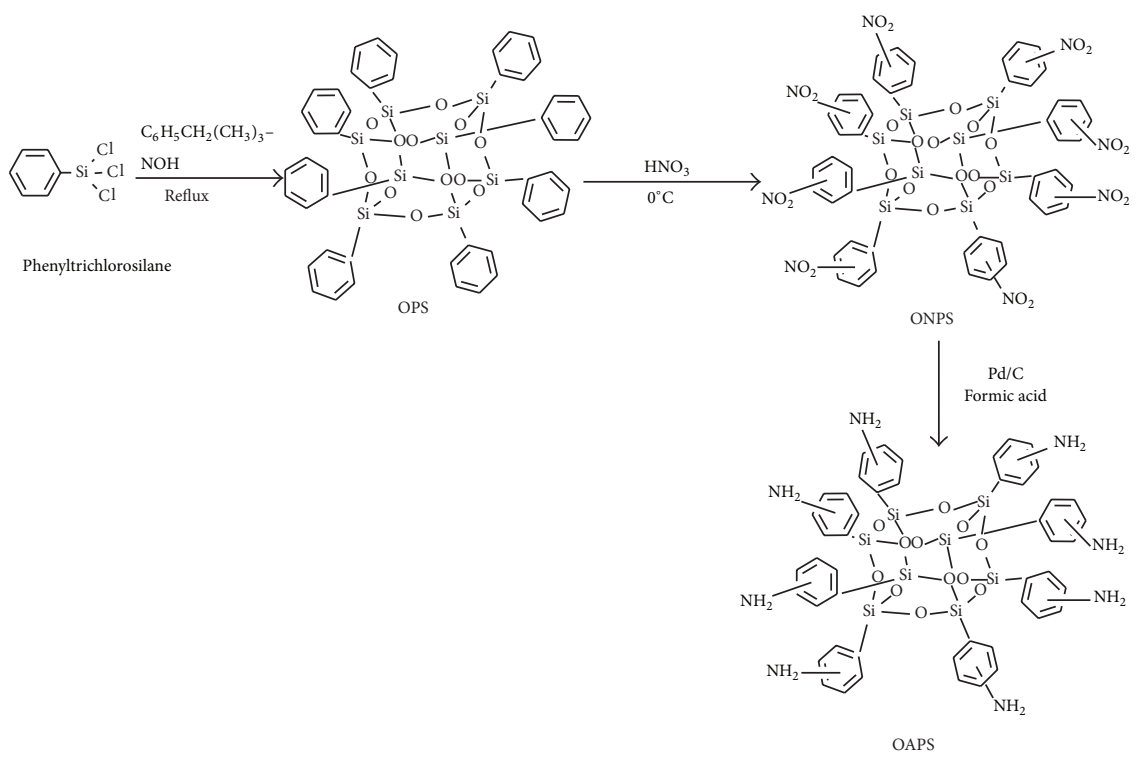

(a)
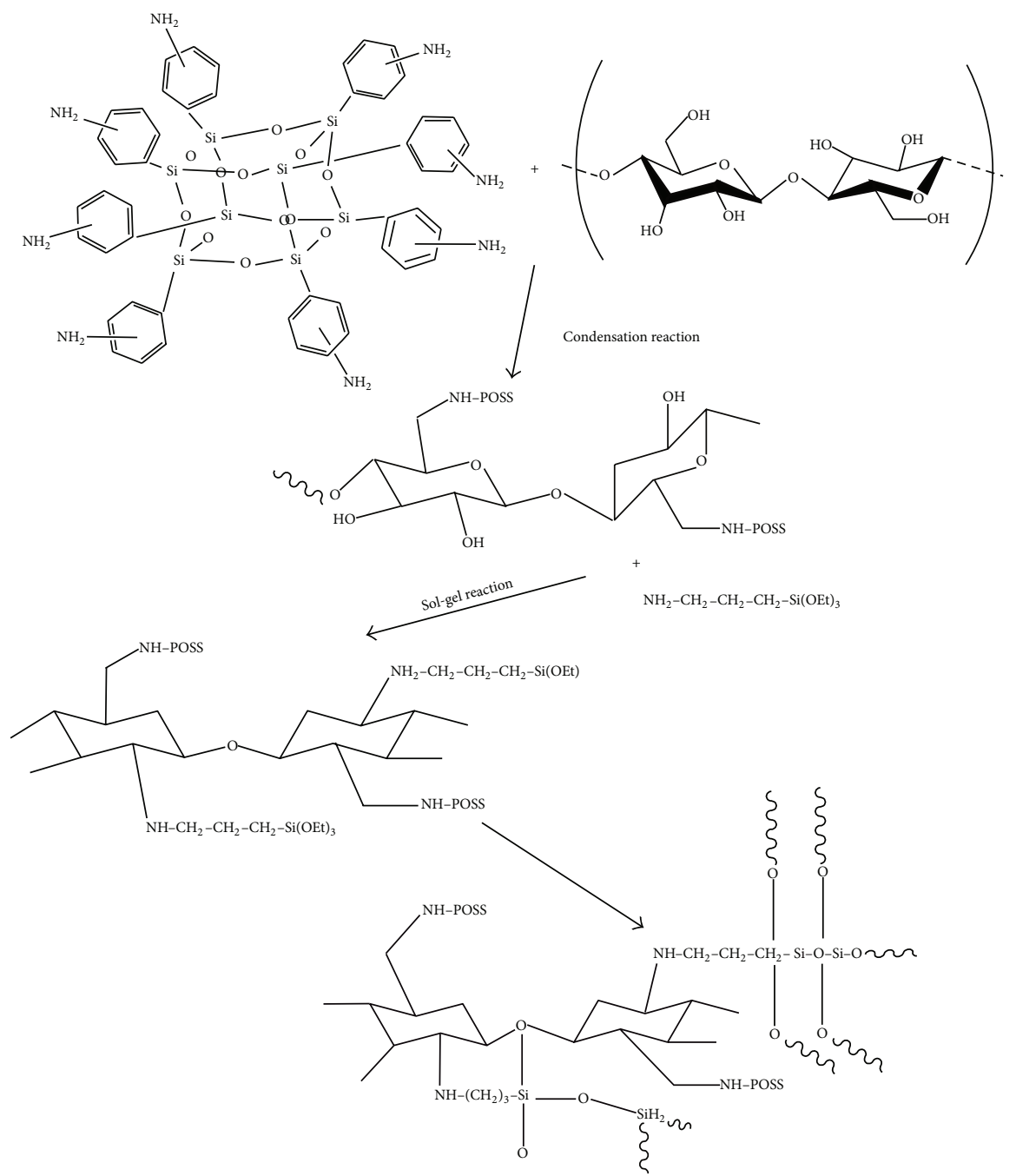

(b)

FIGURE 1: Synthesis schematics of (a) octa(aminophenyl) silsesquioxane (OAPS) and (b) POSS-amine bonded to cellulose-silica hybrid nanocomposites. 
stream of nitrogen gas was passed continuously. The distilled tetrahydrofuran (THF) $(80 \mathrm{~mL})$ and triethylamine were added, the reaction mixture was heated to $60-70^{\circ} \mathrm{C}$, and then $6 \mathrm{~mL}(0.125 \mathrm{~mol})$ of $85 \%$ formic acid was slowly added at $60^{\circ} \mathrm{C}$. After the evolution of carbon dioxide, the solution was separated into two layers and the reaction was carried out at this same temperature for overnight. After that, the reaction mixture was filtered, and $50 \mathrm{~mL}$ of THF and $50 \mathrm{~mL}$ of water were added to the black residue; the mixture was again filtered. Then, $20 \mathrm{~mL}$ of THF and $20 \mathrm{~mL}$ of water were added to dissolve the remaining portion of the black residue, and the mixture was filtered. All the filtrate was mixed together with $50 \mathrm{~mL}$ of ethyl acetate and washed with $100 \mathrm{~mL}$ of water in four times. The organic layer was separated from the aqueous layer with a separating funnel, and the organic layer was kept in $5 \mathrm{~g}$ of magnesium sulfate in the overnight. The organic layer was decanted and precipitated in $2 \mathrm{~L}$ of hexane solvent. The precipitate was collected by filtration, redissolved in $400 \mathrm{~mL}$ of an ethyl acetate/THF mixture $50: 30$ volume ratio $(v / v)$, and precipitated again in $1.5 \mathrm{~L}$ of hexane. The obtained powder was dried in an oven at room temperature.

Synthesis of cellulose-OAPS/SiO 2 hybrid materials was performed by sol-gel crosslinking process. The stoichiometric amount of cellulose $(1.6 \mathrm{~g})$ and the purified form of POSSamine (1.16 g) were dissolved in DMAc solvent and stirred for $1 \mathrm{hr}$ in presence of $\mathrm{HCl}$; then, the mixture was stirred for another $1 \mathrm{hr}$ at $90^{\circ} \mathrm{C}$ condition until the homogeneous solution was obtained. In the same temperature condition, calculated amount of $\gamma$-APTES was added into the homogenous solution and then $10 \mathrm{~g}$ of TEOS, $25 \mathrm{~g}$ of alcohol, $2.5 \mathrm{~g}$ of distilled water, and $\mathrm{HCl}(0.01 \mathrm{M})$ were added and continuously stirred at $90^{\circ} \mathrm{C}$. The resulting solution was a bright yellow transparent color and kept the oven at $90^{\circ} \mathrm{C}$, the reaction mixture was transferred into pet dish and the solvent was allowed to evaporate. Then the glassy cellulose-POSSsilica hybrid nanocomposites are obtained, which is shown in Figure 1(b).

\subsection{Synthesis of Cellulose-POSS-Silica Hybrid Nanocomposites for Antimicrobial Test}

2.3.1. The Agar Diffusion Test. The antimicrobial activity of cellulose-POSS-silica nanoparticles was evaluated against $B$. cereus and $E$. coli. The amount of $0.2 \mathrm{~mL}$ of fresh cultures of organism was inoculated into $5 \mathrm{~mL}$ of sterile Luria Broth and incubated for 3-5 hrs for the standard culture to McFarland standards $(106 \mathrm{CFU} / \mathrm{mL})$. The calculated amount of $100 \mu \mathrm{L}$ of revived culture was added on agar medium and poured on two replicate plates having a diameter of about $8 \mathrm{~mm}$, $50 \mu \mathrm{L}$ samples of cellulose-POSS-silica nanoparticles were added, and $50 \mu \mathrm{L}$ antibiotic (ampicillin) was also added in the separate standard. The Petri plates were incubated at $37^{\circ} \mathrm{C}$ for 20 and 24 hrs.

Colony-Forming Unit. The amount of $100 \mu \mathrm{L}$ of the serial dilution 107 of the culture with and without cellulose-POSSsilica nanoparticles was inoculated on agar plate and was spread over it. The plates were incubated for $20-24 \mathrm{hrs}$ and observed for bacterial growth and viable count per $100 \mu \mathrm{L}$ for a dilution occurred.

\subsubsection{Antimicrobial Test of Cellulose-POSS-Silica Hybrid} Nanomaterial. The stock suspension of cellulose-POSS-silica hybrid nanoparticles with concentrations of $1 \mathrm{mg} / \mathrm{mL}$ was prepared by suspending them in ethanol solution and sonicated for 10 minutes to get the homogenous suspension, for the homogeneous light was kept in UV for $30 \mathrm{~min}$ for sterilization. The overnight culture of the Bacillus's Cereus (F481072) and E. coli (ATCC 35150) was added to Luria Broth with different concentration of silica nanoparticles, respectively, and incubated at $37^{\circ} \mathrm{C}$ for 20 and $24 \mathrm{~h}$ to study the affected microbial activity of hybrid nanoparticles.

2.4. Characterizations. To analyze the synthesis of POSS amine compound, FTIR spectra were recorded for cured samples and ground with solid $\mathrm{KBr}$ by a FTIR spectrometer (Perkin Elmer, RX1) spectrometer. ${ }^{1} \mathrm{H}$ and ${ }^{13} \mathrm{C}$ spectra of the compounds were measured by (TEOL) nuclear magnetic resonance (NMR) machine at $298 \mathrm{~K}$ with tetramethylsilane as an internal standard. Differential scanning calorimetry (DSC, TA Instruments, TA 2000 analyzer) and thermogravimetric analysis (TAG) were used to study the thermal properties of the hybrid nanocomposites. Surface and cross-sectional structure of the nanohybrids were investigated by scanning electron microscopy (SEM, JEOL-JSM model 6360). The cellulose-POSS-silica hybrid samples were further characterized by FTIR spectrum analysis acquired using a Brucker IF5-859 (ATR) mode spectrometer. The structure of hybrid sample was investigated by SEM analysis, X-ray diffraction (XRD, Riguku co $\mathrm{D} / \max -3 \mathrm{C}$ ), and transmission electron microscopy (TEM, Hitachi HT7700).

\section{Result and Discussion}

3.1. Nature of Bonding. The OAPS was synthesized by chemical imidization reactions via an amic acid as an intermediate. The sodium acetate was not used to cyclize the amic acid because the POSS cage structure was not stable in sodium acetate. In this study, the maleic anhydride was added portionwise, whereas OAPS and maleicanhydride were taken together. The stepwise synthesis and characterization of the POSS functionalized materials were executed with FTIR and NMR (not shown in here) in Figure 2(a). The elemental analysis values seem to be agreed with the calculated values of POSS materials. No peak is observed above 9 ppm in the ${ }^{1} \mathrm{H}$ NMR spectrum of ONPS and this confirmed an absence of the dinitro compound. Furthermore, the formation of an orthoisomer is ruled out due to the steric hindrance and possible meta/para isomers which are only feasible. Therefore, the two peaks at 79 and $83.3 \mathrm{ppm}$ in the ${ }^{29} \mathrm{Si}$ NMR spectra of ONPS confirm the existence of the metaand paraisomers, and this further supports the absence of the orthoisomer [25]. The absence of a characteristic $\mathrm{NO}_{2}$ stretching vibration at 1535 and $1347 \mathrm{~cm}^{-1}$ in OAPS indicates the completeness of the nitro reduction. The result indicates that ONPS and OAPS are different from the calculated values because of the differences in the hydrodynamic volume and 


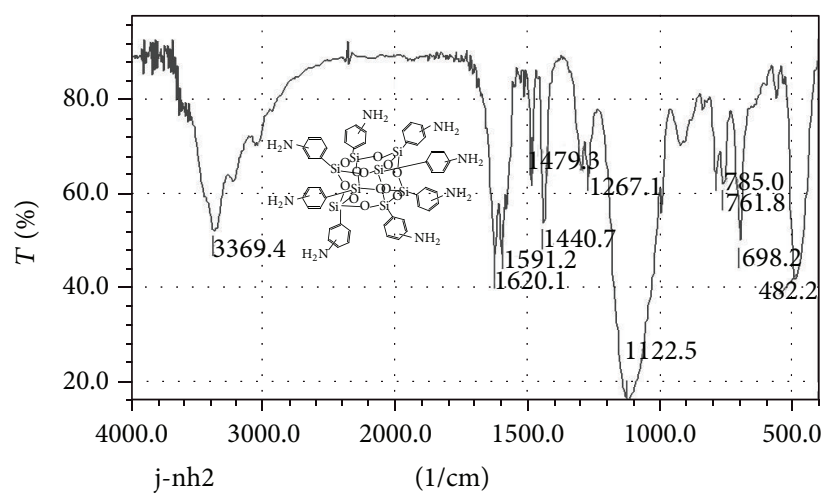

(a)

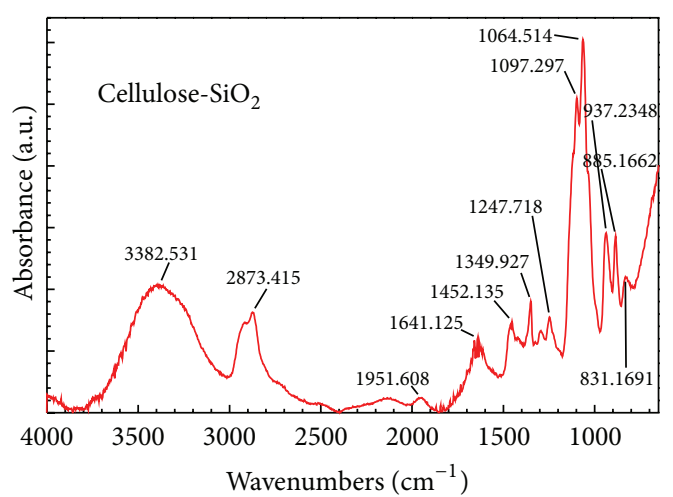

(b)

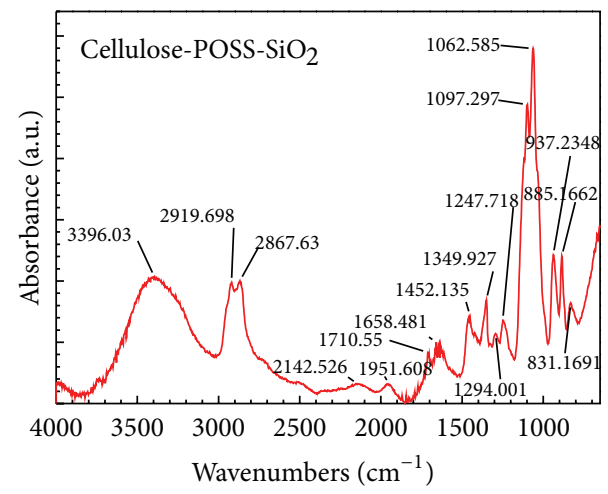

(c)

FIGURE 2: FT-IR spectra of synthesized (a) octa-POSS amine, (b) cellulose-silica, and (c) cellulose-POSS-silica hybrid nanocomposites.

chain flexibility of the polystyrene calibration standards. Moreover, the cellulose-POSS-amine/silica hybrid materials modification was executed by taking FTIR spectra, resulting clearly difference compared to the spectrum of starting material (pulp). In Figure 2(b), the deformation band at $1641 \mathrm{~cm}^{-1}$ confirms the presence of bound water. The bands at 691 and $662 \mathrm{~cm}^{-1}$ were detected in the hybrid material. The third band expected at $1064 \mathrm{~cm}^{-1}$ is overlapped with a broad band between 1000 and $1150 \mathrm{~cm}^{-1}$ which are attributed to O$\mathrm{H}$ bending of primary and secondary alcohol groups of cellulose. Typical bands of silica at the low wavenumber range is associated with rocking, bending (or symmetric stretching), and asymmetric stretching of $\mathrm{SiO}_{2}$ inter-tetrahedral oxygen atoms and symmetrical vibrations at $1064 \mathrm{~cm}^{-1}$ (Si-O-C-) and $1097 \mathrm{~cm}^{-1}(\mathrm{Si}-\mathrm{O}-\mathrm{Si}-)$, which can be confirmed by silica hybrid materials in the cellulose-POSS matrix. Therefore, the POSS amine is bonded to cellulose molecule via coupling agent such as $\gamma$-APTES and TEOS bonded by sol-gel process. In Figure 2(c), FTIR spectrum indicates the clear evidence of crosslinking of cellulose-POSS-silica, which are observed at $3396 \mathrm{~cm}^{-1}$ (for broad NH bonding) and $2919 \mathrm{~cm}^{-1}$ (for C$\mathrm{H}_{2}$ ) starching frequency, $1658 \mathrm{~cm}^{-1}$ (for $\mathrm{CH}$ ), $1062 \mathrm{~cm}^{-1}$ (for $\mathrm{Si}-\mathrm{O}-\mathrm{C}$ ), and $1097 \mathrm{~cm}^{-1}$ (for $\mathrm{Si}-\mathrm{O}-\mathrm{Si}$ ). Therefore, the POSS$\mathrm{NH}_{2}$ bonded to cellulose molecule and also silica from $\gamma$ APTES and TEOS bonded by sol-gel process confirm that the chemical bonding of cellulose-POSS-silica seems to be relatively in good agreement with earlier reports [17].
3.2. Thermal Properties. The DSC and TGA results are shown in Figures 3 and 4, which are for observing the thermal properties of cellulose-POSS-silica hybrid nanocomposites materials. DSC curves of the pure cellulose fiber and grafted polymer nanocomposites were already reported elsewhere $[18,26,27]$. From TGA and the DSC analysis, thermal stability of hybrid materials contains two stages due to the degradation temperature. It is noted that an obvious two different stages may stem from the presence of residual silanol group and secondary amino group present in the cellulosesilica hybrids. In Figure 3, the first and second stages of degradation temperature are also observed by DSC measurement of cellulose, where the curve contains first endothermic peaks at temperatures of $74.1^{\circ} \mathrm{C}, 74.2^{\circ} \mathrm{C}$, and $71.0^{\circ} \mathrm{C}$, respectively. These endothermic peaks are probably associated with the water removal from cellulose and cellulose-POSS/silica hybrid materials. The same effect also studied for suitable temperature between 200 and $400^{\circ} \mathrm{C}$, which corresponds to the scission of the glycosidic bonds with laevoglucose formation. This is due to the fact that thermal degradation reaction starts in the amorphous domain of the cellulosic materials by statistical degradation reaction of cellulose. It was reported that the two the lower peak areas obtained in the DSC plot of areas can explain the decrease of the amount of cationic groups in cellulose chains, which are mainly due to the lower concentration of chemical modifiers. For cellulose, the second endothermic peak initiates at the temperature 


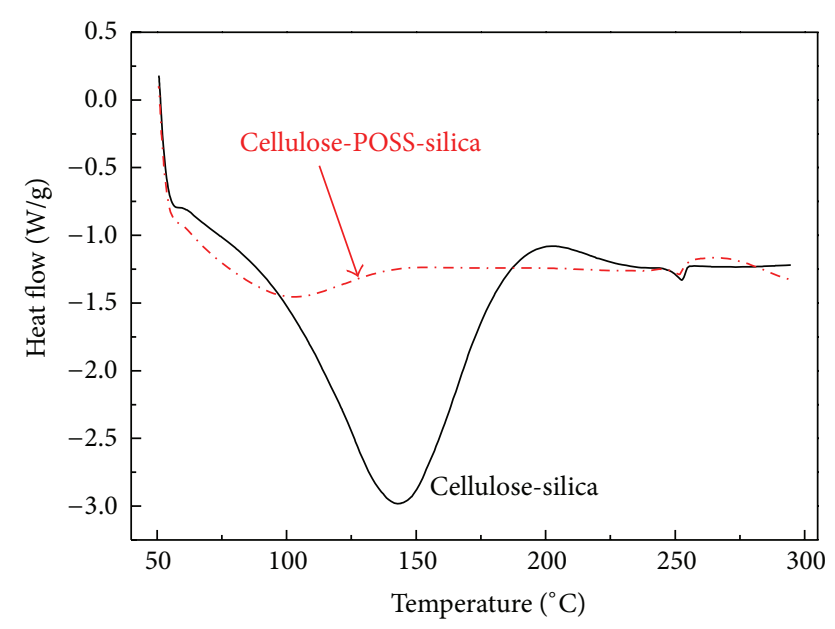

FIGURE 3: Differential scanning calorimetry (DSC) analysis of cellulose-silica and cellulose-POSS-silica hybrid nanocomposites.

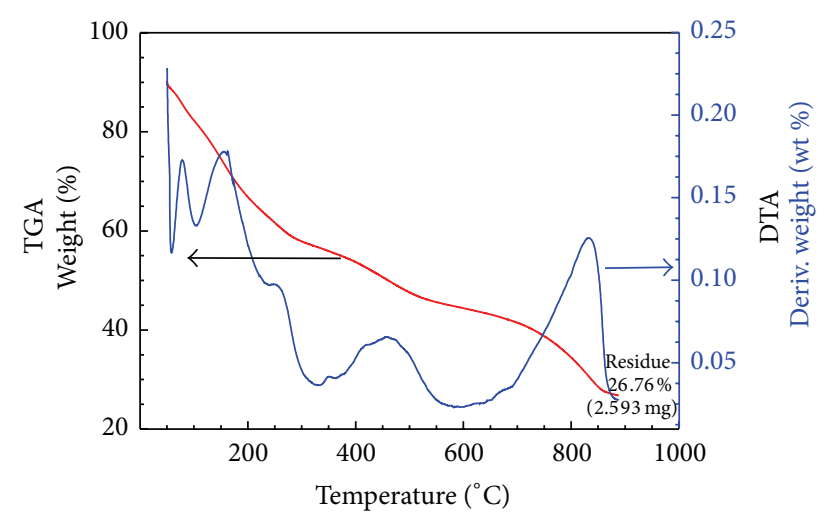

FIgURE 4: Thermogravimetric analysis (TGA) of cellulose-POSSsilica hybrid nanocomposites.

of $340^{\circ} \mathrm{C}$ and finishes at $371^{\circ} \mathrm{C}$. The endothermic change of cellulose obtained in the DSC plot is associated with decomposition processes, which may occur within the fabric during heating process. For cellulose-silica and cellulose-POSS-silica hybrid nanocomposites in Figure 3, the second endothermic peaks initiate at $236^{\circ} \mathrm{C}$ and $219^{\circ} \mathrm{C}$ and finish at $285^{\circ} \mathrm{C}$ and $272^{\circ} \mathrm{C}$, respectively. The endothermic peaks are estimated that there are the interactions between organic components and inorganic components. DSC plots of cellulose/silica hybrids also indicate that the two endothermic peaks are small. Therefore, the endothermic changes obtained in DSC and TGA plots (in Figure 4) may be associated with the decomposition of a part of inorganic/organic hybrids. Moreover, the endothermic peaks are also small. This indicates that the decomposition of cellulose-POSS-amine/silica hybrids may take place in the organic phase. This phenomenon shows that thermal properties of organic/inorganic hybrids can be improved due to the strong interfacial interaction between the organic and inorganic materials. Due to the association with thermal, optical, and mechanical properties, the microphase compatibility has a great influence on morphological changes of the hybrid materials. In order to find the distributions of silica particles prepared via sol-gel process, microstructure of the fractures surface was observed by optical images and SEM analysis.

3.3. Structural Observations of Hybrid Cellulose-POSS-Silica Nanocomposites. The XRD data indicate the influence of POSS nanoparticles on the intensity and structure of patterns. The POSS amine has typical nanosilica hybrid nanocomposites at $7.9^{\circ}$ and $21^{\circ}$ associated with nanohybrid composite structure. In addition, the cellulose-silica hybrid materials are the relative intensity and peak values studied in the cellulose matrix $[18,26,27]$. The main peaks of cellulosePOSS at $14.6^{\circ}, 16.5^{\circ}$, and $22.7^{\circ}$ also associate with cellulose structure and POSS-copolymerized with polymers. This may be due to the decomposition of the amorphous zone during the acid digestion resulting in crystallinity peaks. From the observation of cellulose/silica hybrids, due to the formation without coupling agent, the surface of cellulose-silica crosslinking hybrid seems to be rough. Also the samples show a turbid nature due to the phase separation [28]. However, the surface of cellulose-POSS/silica hybrid fiber with coupling agent is rather smooth as shown in Figure 5. The cellulosePOSS/silica covalent crosslinking hybrid also has heterogeneous structure with well-defined nanostructure. Therefore, the nanoparticles are well dispersed and also form a good surface condition as well as film quality of hybrid materials in Figure 5(c). Similarly, the fractured surfaces of the ONPSmodified an epoxy hybrid system also indicate homogeneous morphology without any apparent phase separation. TEM images of POSS amine and $\gamma$-APTES and TEOS using acid catalyst were also characterized, where the particle size of the hybrids is about $20-100 \mathrm{~nm}$, as shown in Figure 6. This lowest particle size from TEM observations may be due to very strong cohesive interaction between the organic and inorganic materials via coupling agent. The polymer and metal oxide is clearly well dispersed and makes homogeneous structures. The polymer hybrid prepared in the absence of $\gamma$ APTES shows the phase separated turbid coarse product due to nonpossibility of Michael addition reaction, whereas the transparent homogeneous polymer hybrid with particles size is less than $50 \mathrm{~nm}$.

3.4. Optical Transparent Properties. The transparent cellulose-POSS-silica hybrid using Michael addition reaction shows a clear dispersion of the POSS into the silica matrix, that is, molecular level dispersion, where it is clearly seen in the SEM images in Figure 5 and optical image in Figure 7. The hybrid prepared in the absence of $\gamma$-APTES shows phase separated turbid coarse product due to nonpossibility of Michael addition reaction, whereas the transparent homogeneously dispersed POSS-silica with particle sizes less than $1 \mu \mathrm{m}$ is obtained when $\gamma$-APTES is incorporated within the cellulose matrix. Therefore, the $\gamma$-APTES incorporated along with POSS-amine $\mathrm{SiO}_{2}$ is effective combination of Michael addition reaction via sol-gel process. Figure 7 shows the optical images of pure POSS-amine and cellulose-silica hybrid materials are produced with the addition of $0.04 \%$ and $0.02 \%$ of TEOS contents, respectively. From the preparation of POSS-silica and cellulose-POSS-silica hybrid materials, transparent hybrids were obtained in the absence of $\gamma$-APTES. It is found that the $\gamma$-APTES are only active at the 


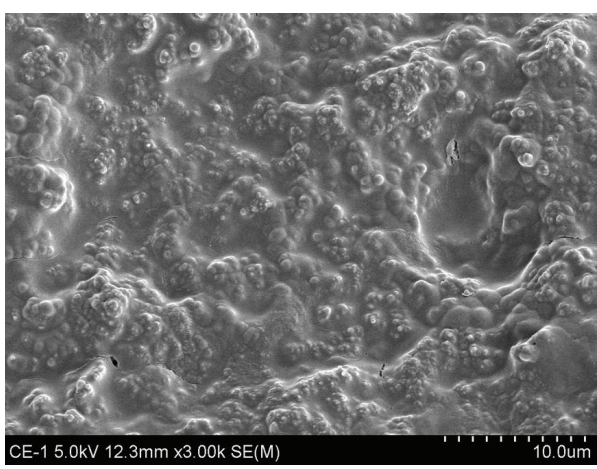

(a)

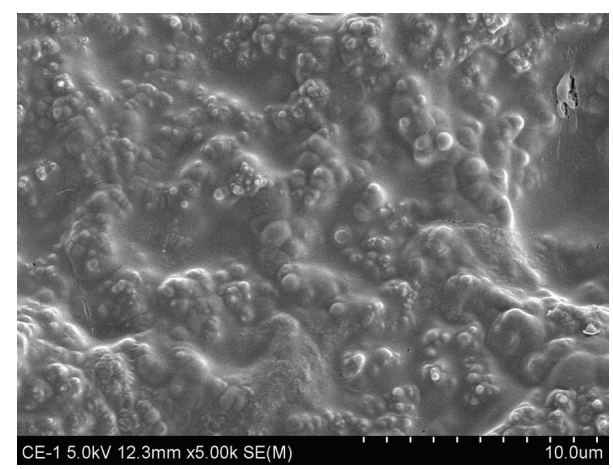

(b)

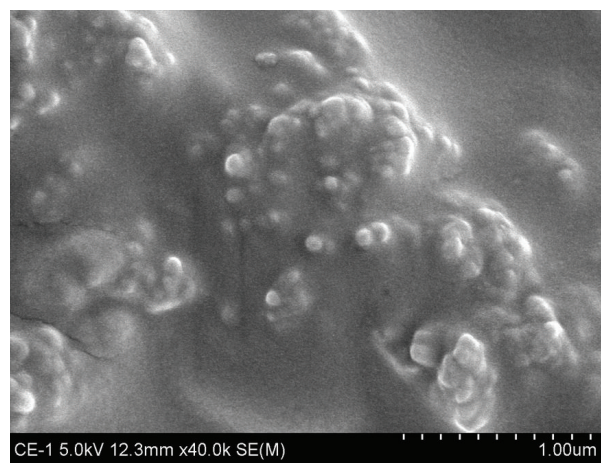

(c)

FIGURE 5: Surface images of cellulose-POSS amine-silica hybrid nanocomposite by SEM measurement with different magnifications.

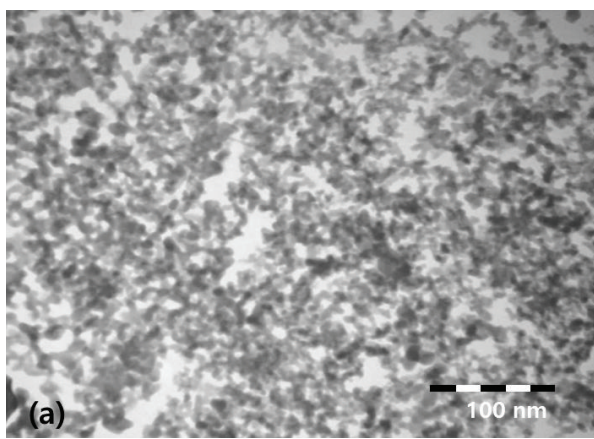

(a)

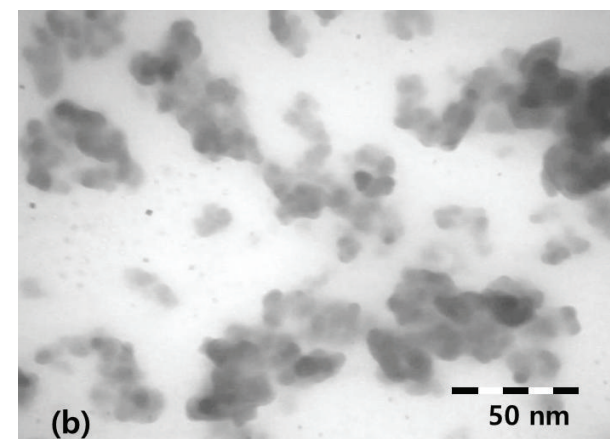

(b)

FIGURE 6: TEM images of (a) cellulose-silica and (b) cellulose-POSS-silica hybrid nanocomposites.

temperature condition above $50^{\circ} \mathrm{C}$, where they can induce the transparent hybrid formation. However, below $50^{\circ} \mathrm{C}$, only turbid materials resulted due to the particles agglomeration [28]. Therefore, the favorable experimental conditions for the preparation of transparent hybrid lie between 60 and $90^{\circ} \mathrm{C}$ in the presence of $\gamma$-APTES. By adapting the above experimental conditions to cellulose-POSS-silica process, organic and inorganic domains sizes can be controlled in the molecular level. However, in microsized reinforcement composites, the refractive indices of reinforcements and resins must be matched at least to the third decimal place to prevent light scattering, which is a critical disadvantage. Even if the refractive indices of resins and reinforcements are accurately matched, the temperature dependency of the resin's refractive index makes the composites opaque or nontransparent as a result of temperature variations.

3.5. Antimicrobial Activity of Hybrid Nanoparticles. Figure 8 shows the population growth of bacteria with increasing concentration of silica nanoparticles to inhibit the Bacillus's cereus and E. coli growth which was confirmed by measuring turbidity. The purpose of plate counting is to estimate the number of cells present based on their ability to give rise to colonies under specific conditions of agar medium, temperature, and time. Both images in Figure 8 show that the number of viable colony decreases from the sample of cellulose-POSS-silica nanoparticles, as compared. For testing the silica nanoparticles susceptibilities of B. cereus and E. coli, 


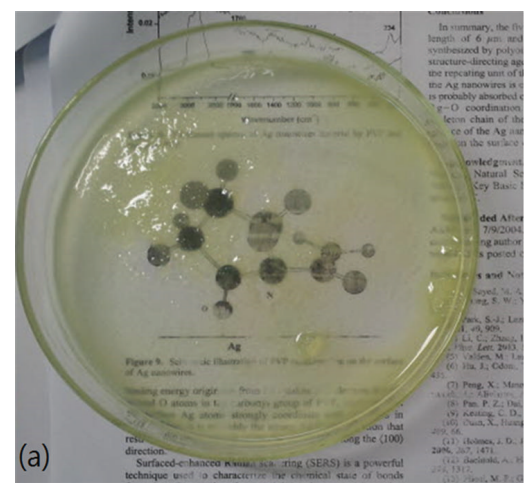

(a)

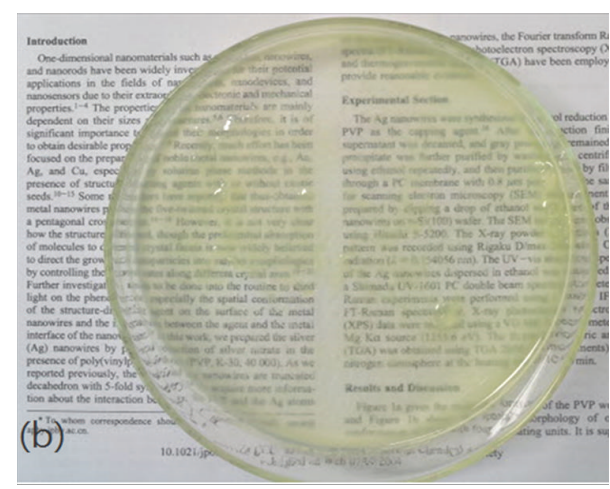

(b)

FIGURE 7: Optically transparent cellulose-POSS-silica hybrid nanocomposite materials. The sol-gel process was performed at the temperature condition of (a) $60^{\circ} \mathrm{C}$ and (b) $90^{\circ} \mathrm{C}$.

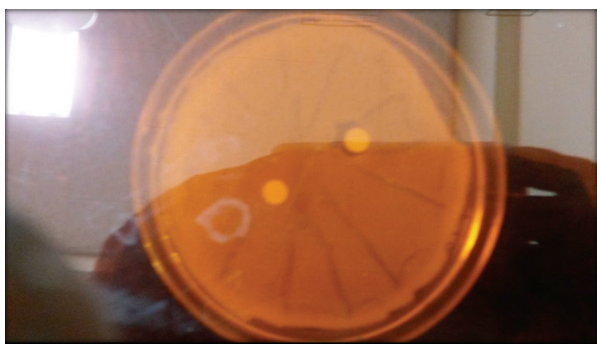

(a)

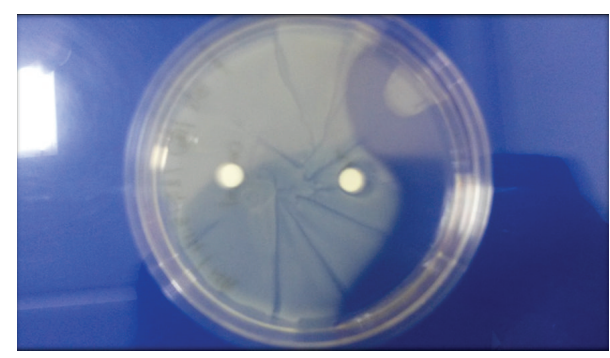

(b)

FIGURE 8: Antimicrobial activity of standard sample (left) versus cellulose-POSS-silica hybrid nanocomposites (right) with (a) B. cereus (+) and (b) E. coli (-) for $24 \mathrm{~h}$ incubation at $37^{\circ} \mathrm{C}$.

we used disc diffusion assay test. As it is evident from the results, cells were highly sensitive to all tested concentration of silica nanoparticles, which was also confirmed from the size of the zone of inhibition. According to several research studies, it is accepted that the metal oxides carry the positive charge and the microorganism contains negative charge, due to the electromagnetic attraction between the microorganism and metal nanooxides. This leads to oxidization and finally death of the microorganism. The silica nanoparticles have been found to be a potent bactericidal compound, although compared to the established antibacterial compound such as ampicillin, but can be studied for superficial application as well as for combinational therapies. The inhibition growth of $E$. coli in the presence and absence of silica nanoparticles has been done, which shows effect of subinhibitory and inhibitory concentration of silica nanoparticle against the both $B$. cereus and $E$. coli till $24 \mathrm{~h}$ of incubation. The antimicrobial effect is greater than $5 \mathrm{~mm}$ and $E$. coli for $8 \mathrm{~mm}$ incubation studies. Therefore, the compression effect of both bacteria is greater for E. coli than for the B. cereus $[29,30]$.

\section{Conclusions}

Organic-inorganic hybrids cellulose-silica incorporated with POSS was synthesized using $\gamma$-APTES as a crosslinking agent through sol-gel method. The cellulose-silica and cellulosePOSS-silica were developed in presence of $\gamma$-APTES hybrids from new molecular structures containing POSS nanosilica particles. The POSS amine particles are well dispersed into cellulose host matrix by bonding through covalent bonds with nanometer scale. The thermal properties of the POSS amine bonded to cellulose hybrids are greatly improved. The homogeneity was influenced by the rate of gelation and hydrolysis of alkoxysilanes and also the occurrence of Michael addition reaction between the amino group groups. At above $90^{\circ} \mathrm{C}$, cellulose-POSS amine and silica are mixed homogeneously by crosslinking reaction between the cellulose-POSS-amine and inorganic oxides through covalent bond. The cellulose-POSS-silica hybrid materials were greatly improved by obtaining optical transparency, thermal stability, and antimicrobial effect against the pathogenic bacteria. Therefore, the cellulose-POSS-silica hybrid materials may find wide applications such as optoelectronics, sensor, biosensor, and also medical applications.

\section{Conflict of Interests}

The authors declare that there is no conflict of interests regarding the publication of this paper.

\section{Acknowledgments}

This research work was supported by Basic Science Research Program (20120004034), Agency of Defense and Development Program (ADD CBDRC, CBD-12), and pressure 
monitoring and redesignable medical smart wear (10044722) project through KIAT.

\section{References}

[1] G. Zheng, Y. Cui, E. Karabulut, L. Wågberg, H. Zhu, and L. $\mathrm{Hu}$, "Nanostructured paper for flexible energy and electronic devices," MRS Bulletin, vol. 38, no. 4, pp. 320-325, 2013.

[2] R. J. Moon, A. Martini, J. Nairn, J. Simonsen, and J. Youngblood, "Cellulose nanomaterials review: structure, properties and nanocomposites," Chemical Society Reviews, vol. 40, no. 7, pp. 3941-3994, 2011.

[3] D. Tobjörk and R. Österbacka, "Paper electronics," Advanced Materials, vol. 23, no. 17, pp. 1935-1961, 2011.

[4] L. Nyholm, G. Nyström, A. Mihranyan, and M. Strømme, "Toward flexible polymer and paper-based energy storage devices," Advanced Materials, vol. 23, no. 33, pp. 3751-3769, 2011.

[5] E. Sjostrom, Wood Chemistry-Fundamentals and Applications, Academic Press, San Diego, Calif, USA, 1993.

[6] V. D. Athawale and V. Lele, "Graft copolymerization onto starch. II. Grafting of acrylic acid and preparation of it's hydrogels," Carbohydrate Polymers, vol. 35, no. 1-2, pp. 21-27, 1998.

[7] J. P. Ayers, D. W. Greve, and I. J. Oppenheim, "Energy scavenging for sensor applications using structural strains," in Smart Structures and Materials 2003: Smart Systems and Nondestructive Evaluation for Civil Infrastructures, vol. 5057 of Proceedings of SPIE, pp. 364-375, San Diego, Calif, USA, August 2003.

[8] M. P. Houng, W. L. Lu, T. H. Yang, and K.-W. Lee, "Characterization of the nanoporous template using anodic alumina method," Journal of Nanomaterials, vol. 2014, Article ID 130716, 7 pages, 2014.

[9] F. Bauer, U. Decker, H. Ernst et al., "Functionalized inorganic/organic nanocomposites as new basic raw materials for adhesives and sealants part 2," International Journal of Adhesion and Adhesives, vol. 26, no. 7, pp. 567-570, 2006.

[10] S. L. Burkett, S. D. Sims, and S. Mann, "Synthesis of hybrid inorganic-organic mesoporous silica by co-condensation of siloxane and organosilane precursors-first example of covalently linked, ordered hybrid inorganic-organic networks," Chemical Communications, vol. 48, no. 70, pp. 1366-1368, 1996.

[11] Y. T. Chang, C. F. Shu, C. M. Leu, and K. H. Wei, "Synthesis and characterization of hyperbranched aromatic poly(ether imide)s with terminal amino groups," Journal of Polymer Science. Part A: Polymer Chemistry, vol. 41, no. 23, pp. 3726-3736, 2003.

[12] J. Choi, J. Harcup, A. F. Yee, Q. Zhu, and R. M. Laine, "Organic/inorganic hybrid composites from cubic silsesquioxanes," Journal of the American Chemical Society, vol. 123, no. 46, pp. 11420-11430, 2001.

[13] J. Choi, A. F. Yee, and R. M. Laine, "Toughening of cubic silsesquioxane epoxy nanocomposites using core-shell rubber particles: a three-component hybrid system," Macromolecules, vol. 37, no. 9, pp. 3267-3276, 2004.

[14] G. Cardoen and E. B. Coughlin, "Hemi-telechelic polystyrenePOSS copolymers as model systems for the study of welldefined inorganic/organic hybrid materials," Macromolecules, vol. 37, no. 13, pp. 5123-5126, 2004.

[15] A. Fina, D. Tabuani, A. Frache, and G. Camino, "Polypropylenepolyhedral oligomeric silsesquioxanes (POSS) nanocomposites," Polymer, vol. 46, no. 19, pp. 7855-7866, 2005.
[16] S. Yun, J. Kim, and K. S. Lee, "Evaluation of cellulose electroactive paper made by tape casting and zone stretching methods," International Journal of Precision Engineering and Manufacturing, vol. 11, no. 6, pp. 987-990, 2010.

[17] A. Hou, Y. Shi, and Y. Yu, "Preparation of the cellulose/silica hybrid containing cationic group by sol-gel crosslinking process and its dyeing properties," Carbohydrate Polymers, vol. 77, no. 2, pp. 201-205, 2009.

[18] A. Hou, X. Wang, and L. Wu, "Effect of microwave irradiation on the physical properties and morphological structures of cotton cellulose," Carbohydrate Polymers, vol. 74, no. 4, pp. 934937, 2008.

[19] P. Innocenzi and B. Lebeau, "Organic-inorganic hybrid materials for non-linear optics," Journal of Materials Chemistry, vol. 15, no. 35-36, pp. 3821-3831, 2005.

[20] B. J. Jin, S. H. Bae, S. Y. Lee, and S. Im, "Effects of native defects on optical and electrical properties of $\mathrm{ZnO}$ prepared by pulsed laser deposition," Materials Science and Engineering B, vol. 71, no. 1, pp. 301-305, 2000.

[21] K. D. Kim and H. T. Kim, "Formation of silica nanoparticles by hydrolysis of TEOS using a mixed semi-batch/batch method," Journal of Sol-Gel Science and Technology, vol. 25, no. 3, pp. 183189, 2002.

[22] R. M. Laine, J. Choi, and I. Lee, "Organic-inorganic nanocomposites with completely defined interfacial interactions," Advanced Materials, vol. 13, no. 11, pp. 800-803, 2001.

[23] C. Sanchez, B. Julián, P. Belleville, and M. Popall, "Applications of hybrid organic-inorganic nanocomposites," Journal of Materials Chemistry, vol. 15, no. 35-36, pp. 3559-3592, 2005.

[24] F. Von Nussbaum, M. Brands, B. Hinzen, S. Weigand, and D. Häbich, "Antibacterial natural products in medicinal chemistry-exodus or revival?" Angewandte Chemie: International Edition, vol. 45, no. 31, pp. 5072-5129, 2006.

[25] R. Tamaki, Y. Tanaka, M. Z. Asuncion, J. Choi, and R. M. Laine, "Octa(aminophenyl)silsesquioxane as a nanoconstruction site," Journal of the American Chemical Society, vol. 123, no. 49, pp. 12416-12417, 2001.

[26] D. M. Fox, P. H. Maupin, R. H. Harris Jr. et al., "Use of a polyhedral oligomeric silsesquioxane (POSS)-imidazolium cation as an organic modifier for montmorillonite," Langmuir, vol. 23, no. 14, pp. 7707-7714, 2007.

[27] D. Ciolacu, F. Ciolacu, and V. I. Popa, "Amorphous cellulosestructure and characterization," Cellulose Chemistry and Technology, vol. 45, no. 1-2, pp. 13-21, 2011.

[28] S. K. Park, K. D. Kim, and H. T. Kim, "Preparation of silica nanoparticles: determination of the optimal synthesis conditions for small and uniform particles," Colloids and Surfaces A: Physicochemical and Engineering Aspects, vol. 197, no. 1-3, pp. 7-17, 2002.

[29] H. Nabeshi, T. Yoshikawa, K. Matsuyama et al., "Systemic distribution, nuclear entry and cytotoxicity of amorphous nanosilica following topical application," Biomaterials, vol. 32, no. 11, pp. 2713-2724, 2011.

[30] N. Agata, M. Mori, M. Ohta, S. Suwan, I. Ohtani, and M. Isobe, "A novel dodecadepsipeptide, cereulide, isolated from Bacillus cereus causes vacuole formation in HEp-2 cells," FEMS Microbiology Letters, vol. 121, no. 1, pp. 31-34, 1994. 

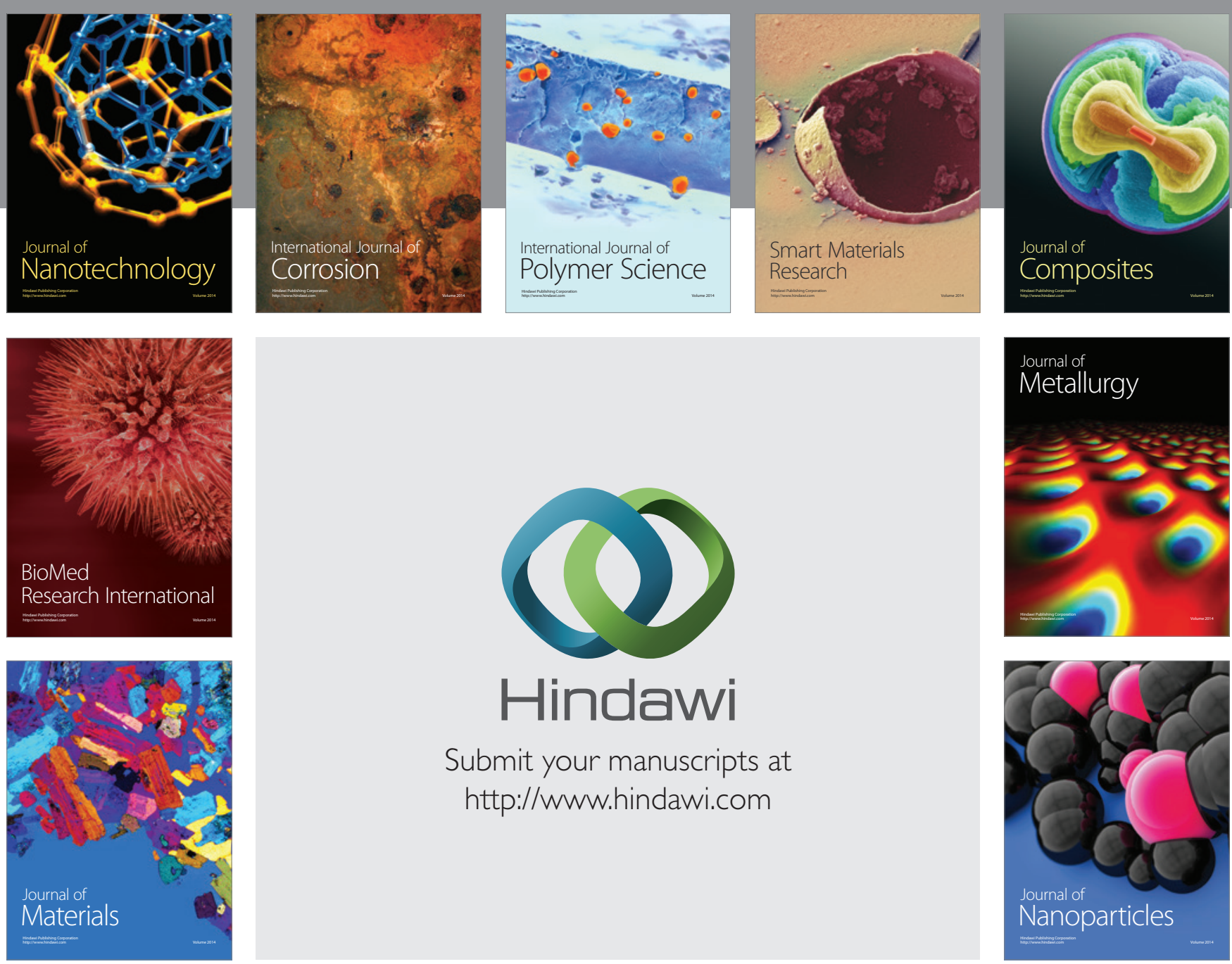

Submit your manuscripts at http://www.hindawi.com
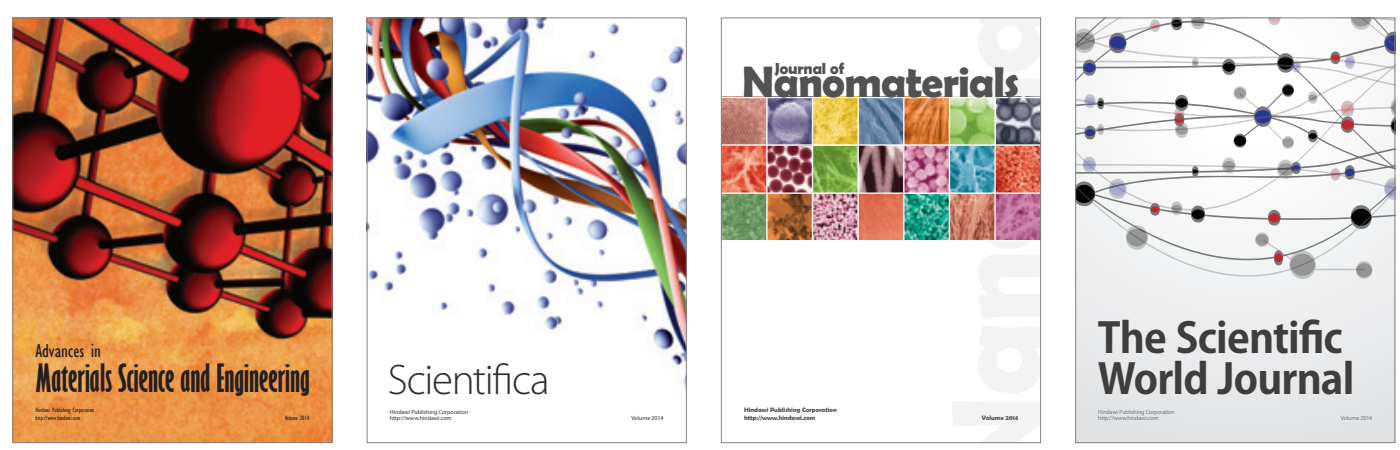

\section{The Scientific World Journal}
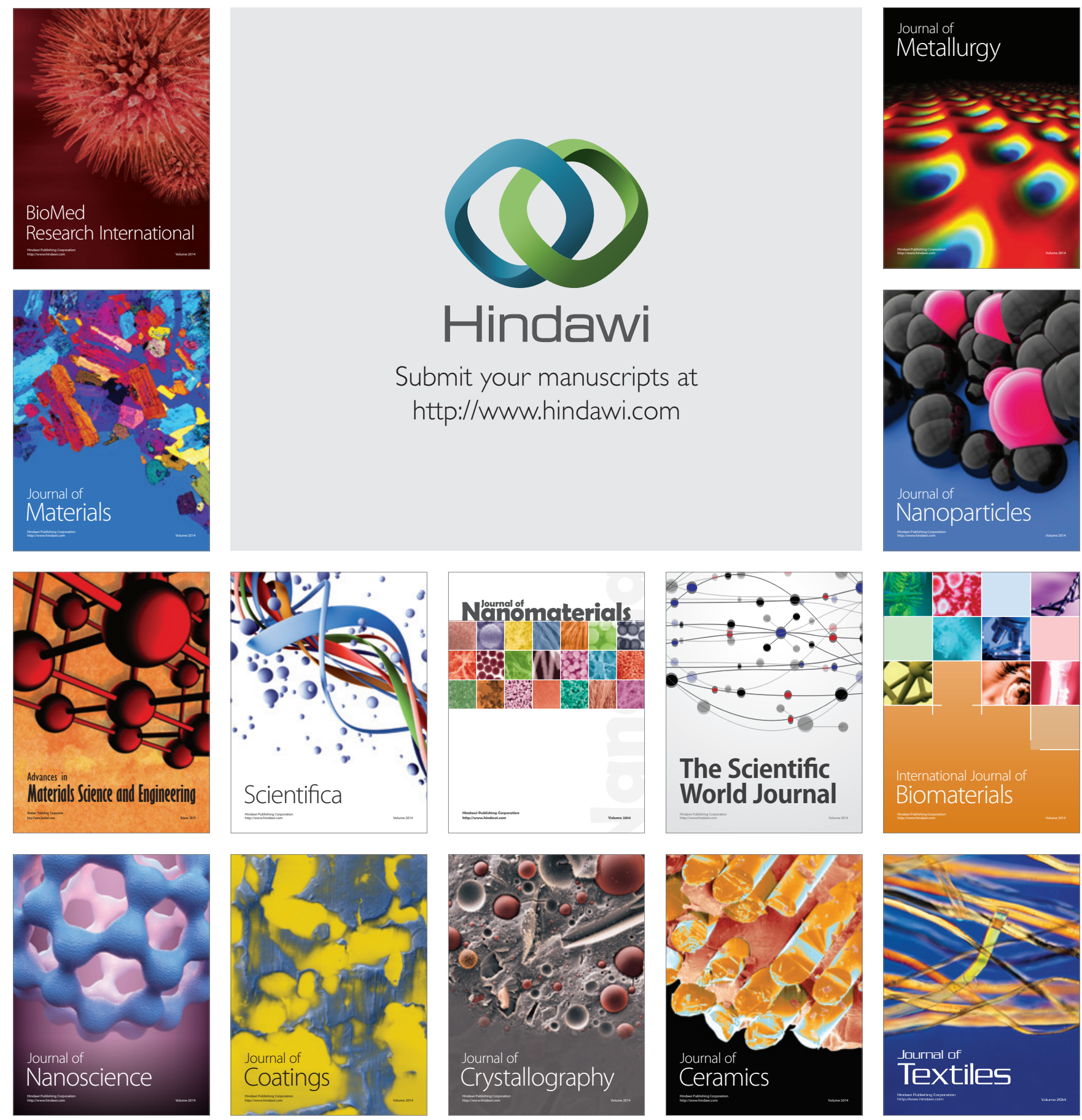